\title{
artigo
}

\section{Ciranda entre Educação e Saúde: Aspectos da Saúde Mental do Adolescente em Contexto Escolar em Tempos de Pandemia}

\author{
Ciranda between Education and Health: Aspects of Adolescent Mental Health in School Context in Times of Pandemic \\ Ciranda entre educación y salud: aspectos de la salud mental de los adolescentes en el contexto escolar en tiempos de \\ pandemia
}

\begin{abstract}
RESUMO
O contexto escolar e o público adolescente foram bastante impactados pelo novo cenário de pandemia. Objetivo: Analisar quais fatores decorrentes da pandemia tem repercutido na saúde mental dos adolescentes em contexto escolar, bem como averiguar as principais estratégias promotoras de saúde mental no contexto escolar em tempos de pandemia. Método: Trata-se de um relato de experiência de práticas multiprofissionais no contexto escolar, envolvendo as áreas da psicologia e da enfermagem com os alunos do ensino médio de uma escola filantrópica da cidade de Teresina - PI. Resultados: Foram identificadas demandas relacionadas à saúde do adolescente e a partir das práticas dos respectivos profissionais foi possível oportunizar estratégias de promoção à saúde dos mesmos. Conclusão: Ficou evidente que mesmo diante da situação pandêmica e das adversidades, a preocupação com a saúde e bem estar dos alunos é única e impulsionou para o desenvolvimento de novas ações dentro da escola. DESCRITORES: Adolescente; Saúde Mental; Infecções por coronavírus; Instituições acadêmicas.
\end{abstract}

\section{ABSTRACT}

The school context and adolescent public were greatly impacted by the new pandemic scenario. Objective: To analyze which factors resulting from the pandemic have had an impact on the mental health of adolescents in the school context, as well as to investigate the main strategies that promote mental health in the school context in times of pandemic. Method: This is an experience report of multiprofessional practices in the school context, involving the areas of psychology and nursing with high school students from a philanthropic school in the city of Teresina - PI. Results: Demands related to adolescent health were identified and, based on the practices of the respective professionals, it was possible to create strategies to promote their health. Conclusion: It was evident that even in the face of the pandemic situation and the adversities, the concern with the students' health and well-being is unique and boosted the development of new actions within the school.

DESCRIPTORS: Adolescent; Mental health; Coronavirus infections; Academic institutions.

\section{RESUMEN}

El contexto escolar y el público adolescente se vieron muya fectados por el nuevo escenario pandémico. Objetivo: Analizar qué factores resultantes de la pandemia han impactado en la salud mental de los adolescentes en el contexto escolar, así como conocer las principales estrategias que promuev en la salud mental en el contexto escolar en tiempos de pandemia. Método: Se trata de un relato de experiencia de prácticas multiprofesional esenel contexto escolar, involucrando las áreas de psicología y enfermería con estudiantes de secundaria de una escuela filantrópica de la ciudad de Teresina - PI. Resultados: Se identificar on las demandas relacionadas con las alud de los adolescentes y, a partir de las prácticas de los respectivos profesionales, fue posible generar estrategias para promover su salud. Conclusión: Se evidenció que aún frente a la situación pandémica y las adversidades, la preocupación por la salud y el bienestar de los estudiantes es única e impulsó el desarrollo de nuevas acciones dentro de la escuela.

DESCRIPTORES: Adolescente; Salud mental; Infecciones por coronavirus; Instituciones académicas.

RECEBIDO EM: 30/03/2021 APROVADO EM: 22/04/2021 


\section{Ana Caroline Inácio de Alencar}

Psicóloga Escolar Educacional. Instituto Dom Barreto. Pós-graduanda em Psicologia Escolar Educacional (UNIFSA) e pós-graduanda em Suicidologia(FAEVE). Atualmente é psicóloga escolar do Instituto Dom Barreto - Escola Popular Madre Maria Villac. ORCID: 0000-0002-4275-587X

\section{Delite Conceição Rocha Barros Lemos}

Mestre em Ciências da Educação, Especialização em Docência Superior, Educação, Saúde Mental, Educação e Gestalt Terapia. Atua como Psicóloga na Fundação Municipal de Saúde no Município de Teresina Piauí, Psicóloga Escolar Educacional no Instituto Dom Barreto. Coordenadora do Projeto Bordando Histórias de Vida.

ORCID: 0000-0001-5800-2840

\section{Érica Vanessa Rodrigues da Silva}

Graduada em Psicologia pela Universidade Estadual do Piauí. Especialista em Psicologia Escolar Educacional e Pós-graduanda em Educação Infantil. Atua como Psicóloga Escolar Educacional no Instituto Dom Barreto - Escola Madre Maria Villac e Docente no Ensino Superior (Faespi). Membro da Associação Brasileira de Psicologia Escolar (Representação Piauí).

ORCID: 0000-0003-1283-374X

\section{Estefane Vasconcelos Sousa}

Enfermeira. Formada pelo Centro de ensino Unificado de Teresina - CEUT. Atua como enfermeira do Instituto Dom Barreto. ORCID: 0000-0001-5292-3876

\section{Kaynna Beatriz do Nascimento Sousa}

Psicóloga formada pela instituição UniFacid|Wyden, e Pós-graduanda em Neuropsicologia pela IPOG. Atua na área clínica, com atendimentos para crianças, adolescentes e adultos.

ORCID: 0000-0001-7340-7029

\section{Paulo César Borges de Sousa Filho}

Mestrando em Biotecnologia e Atenção Básica de Saúde, Esp.em Avaliação Psicológica e Psicologia Escolar Educacional. Formação em ACP. Psicólogo Escolar Educacional pelo IDB e docente do curso de Psicologia e Odontologia pela Unifacid.

ORCID: 0000-0001-8198-300X

\section{INTRODUÇÃO}

A escola se configura em um espaço de interação social, que promove educação, construção de valores, formação cidadã, bem como do desenvolvimento de habilidades socioemocionais e autoconhecimento. É nesse organismo vivo que se pode perceber o tecer diário na formação do sujeito, aos poucos desenhado através do pensamento crítico, da aquisição de conhecimentos e da expressividade emocional.

A Base Nacional Comum Curricular compreende que o processo educativo vai além do conhecimento e da propagação científica, sendo necessário o desenvolvimento de competências e habilidades, que contribuam na formação de atitudes e valores dos indivíduos inseridos dentro da comunidade escolar. Em função disso, a BNCC propõe na sua oitava competência um relevante aspecto para a promoção da saúde física e mental do sujeito: Conhecer-se, apreciar-se e cuidar de sua saúde física e emocional, compreendendo-se na diversidade humana e reconhecendo suas emoções e as dos outros, com autocrítica e capacidade para lidar com elas ${ }^{1}$.

Diante do contexto apresentado, entende-se que há uma ciranda ${ }^{1}$ entre escola, educação e saúde, compreendendo que quanto maisocorre a comunicação entre esses elementos, mais benefícios poderão ser percebidos na comunidade escolar, indo assim de encontro à perspectiva do desenvolvimento integral do aluno proposto na BNCC.

Os bons níveis de educação, que envolvem a melhor compreensão dos conteúdos, podem ser percebidos em indivíduos mais saudáveis ${ }^{2}$. Em função disso, a escola deve ser entendida como promotora de saúde, através de práticas interdisciplinares e multiprofissionais, intervindo nos mais diversos contextos de vida do estudante.
Nessa perspectiva, as metodologias educacionais devem priorizar a participação e a interação dos atores da escola, compreendendo que o conceito de saúde configura um conjunto de habilidades, tais como autonomia e a tomada de decisões, sendo necessário estimulá-las nos indivíduos, favorecendo atitudes mais saudáveis como a corresponsabilização e o enfrentamento das situações ${ }^{3}$

Dessa forma, diante do cenário pandêmico em que o contexto escolar está vivenciando com completa mudança da rotina e estrutura vivenciada até então, o presente estudo torna-se relevante à medida que busca compreender os diversos fatores que interferem na saúde mental dos adolescentes, fazendo um resgate da relação essencial entre saúde e escola, levando em consideração os estágios de desenvolvimento humano e o processo de ensino-aprendizagem de cada indivíduo. 
Em virtude disto, as práticas escolares evidenciaram a necessidade de buscar novos aportes teóricos para ampliar o conhecimento e diversificar as intervenções que possam colaborar significativamente para o fortalecimento desse sujeito no que se refere ao enfrentamento dos desafios impostos pela pandemia do novo coronavírus (SARS-CoV-2) no contexto escolar. Dentre esses desafios, encontramos o início das atividades on-line antes pouco frequente na escola, como acesso a vídeos educativos em plataformas digitais, atividades on-line síncronas e assíncronas, realização de testes on-line e utilização de mídias sociais de longo alcance, como WhatsApp. Tais mudanças foram norteadas pelo Ministério da Educação (MEC) e o Conselho Nacional de Educação (CNE).

Diante desta ótica, este estudo tem como objetivo geral analisar quais fatores decorrentes da pandemia tem repercutido na saúde mental do adolescente em contexto escolar, apresentando os seguintes objetivos específicos: investigar o impacto da pandemia do novo coronavírus sobre a saúde mental dos adolescentes e averiguar as principais estratégias promotoras de saúde mental no contexto escolar em tempos de pandemia.

\section{MÉTOdOS}

O respectivo trabalho trata-se de um relato de experiência na área da psicologia escolar educacional após o início da pandemia (Covid-19) no contexto do ensino médio em uma escola filantrópica na cidade de Teresina/PI. O trabalho possui uma abordagem qualitativa, na qual o cientista é ao mesmo tempo o sujeito e o objeto de suas pesquisas. Tendo assim, a natureza de uma pesquisa aplicada, a qual objetiva gerar conhecimentos para aplicação prática, dirigidos à solução de problemas específicos. Possui também objetivo explicativo, pois explica o porquê das coisas através dos resultados oferecidos ${ }^{4}$.

Foram incluídas no relato de experiência as três turmas de cada série do segmento já mencionado, que funcionam no período vespertino, totalizando uma média de cem alunos. Não fazendo parte do respectivo relato as práticas nas séries da Educação Infantil, Fundamental anos iniciais e anos finais. O relato de experiência abarca o período letivo de janeiro de 2020 (início da pandemia) a março de 2021 (contexto pandêmico atual). No início de abril de 2020, quando teve início a pandemia, as aulas foram ministradas de forma assíncrona e posteriormente, aconteceram através da plataforma Google Meet. No ano de 2021, houve uma transição no formato dessas aulas sendo estas ofertadas no modelo híbrido.

\section{Em virtude disto,} uma das múltiplas funçôes do psicólogo escolar é de agente mediador de reflexão e conscientização das relações existentes

\section{neste contexto.}

Os encontros em sala com a equipe de psicologia ocorrem uma vez por semana e tem duração de 45 minutos. Os recursos utilizados como subsídio para a discussão das temáticas em sala, são: slides, vídeos e dinâmicas. Para respaldar as intervenções, são utilizados referenciais teóricos que contemplam as demandas vivenciadas, encontradas em artigos periódicos, no formato PDF e de acesso livre, bem como livros impressos da referida área, sendo estes: O Poder do Hábito (Charles Duhigg); A Coragem de ser Imperfeito (Brené Brown); Inteligência Emocional: a teoria revolucionária que redefine o que é ser inteligente (Daniel Goleman); Foco: atenção e seu papel fundamental para o sucesso (Daniel Goleman); O Caminho da Autotransformação (Eva Pierrakos) e Sobre a Morte e o Morrer (Elisabeth Kubler-Ross). É importante ressaltar que as intervenções realizadas em sala de aula continuam em andamento.

Desse modo, foram relatadas as principais práticas realizadas pelo serviço de psicologia e enfermagem na escola neste intervalo de tempo e nestas séries mencionadas. Sendo realizado posterior diálogo com os estudos que versam sobre as práticas descritas de forma categorizada.

\section{RESULTADOS}

\section{O fazer no ensino médio}

A Psicologia Escolar define-se como uma área de atuação da Psicologia que, dentre outras atribuições, assume um compromisso teórico e prático com as questôes relativas à escola e a seus processos, sua dinâmica, resultados e atores ${ }^{5}$. Em virtude disto, uma das múltiplas funções do psicólogo escolar é de agente mediador de reflexão e conscientização das relações existentes neste contexto.

Portanto, dada a explanação, o setor de psicologia na escola funciona há treze anos, realizando práticas com toda a comunidade. Estas ações envolvem intervenções a nível preventivo e remediativo a partir das demandas que emergem no cotidiano. No ensino médio, as principais estratégias desenvolvidas pelo PEE (Psicólogo Escolar Educacional) estão relacionadas ao desenvolvimento de um programa de Inteligência Socioemocional nas séries do $1^{\circ}$ e $2^{\circ}$ ano do respectivo segmento. $\mathrm{Na}$ turma de $3^{\circ}$ ano, são desenvolvidas atividades relacionadas ao projeto intitulado "Orientação Profissional Vocacional" (OPV), tendo como objetivo oferecer um espaço de apoio e expressão que contribua para o fortalecimento deste jovem diante das demandas presentes no seu processo de construção da identidade profissional.

Além dessas práticas já mencionadas, são oferecidas palestras mensais denominadas "Histórias Inspiradoras" que tem como intuito motivar os alunos através dos relatos 
de superação dos convidados diante dos desafios enfrentados ao longo da sua vida, que servem como aprendizado para estes jovens. Outrossim, também acontecem palestras intituladas "Mesa Redonda das Profissões", que são momentos de esclarecimento a respeito das profissões de interesse dos alunos, bem como uma atualização das mudanças que estão acontecendo nas profissões no contexto pandêmico. Sobre este último, essas palestras fazem parte das atividades relacionadas ao projeto de "Orientação Profissional Vocacional" acima citado, desenvolvendo atividades que favoreçam o reconhecimento dos potenciais internos do jovem, além de facilitar uma compreensão a respeito das suas habilidades associadas à escolha profissional.

Ocorrem também acompanhamentos individuais aos alunos e famílias, além da produção de relatórios destes, quando solicitados por profissionais externos. Estas atividades articuladas ao projeto de vida dos adolescentes, contribuem para o desenvolvimento de um repertório emocional de enfrentamento, tendo em vista o foco nas capacidades e competências que o jovem possui para trilhar o caminho da sua autorrealização pessoal e profissional, tendo sido essenciais para o enfrentamento das adversidades percebidas decorrentes das transformações ocasionadas pela COVID-19. Fica então perceptível que algumas práticas já realizadas antes da pandemia, se mantiveram, porém com as novas demandas, novas práticas precisaram compor o fazer do PEE no ensino médio.

\section{Demandas relacionadas à saúde mental dos estudantes durante a pandemia}

No dia 16 de março de 2020, houve a suspensão das aulas no estado do Piauí, em virtude do novo coronavírus (SARS-CoV-2). Após o período de férias coletivas, as aulas retornaram em abril, a princípio com aulas assíncronas e posteriormente no modelo de ensino híbrido. Desde esse período até o momento atual, foi observado um aumento nas demandas relacionadas à saúde mental dos adolescentes, dentre elas: medo, ansiedade, luto, alteração no sono, na alimentação, desmotivação e instabilidade emocional. As demandas supracitadas foram identificadas de três formas com os alunos do ensino médio: através de um diagnóstico socioemocional, realizado por meio de um questionário on-line, além da busca ativa dos alunos ao Serviço de Psicologia e durante as aulas realizadas onde os alunos puderam expressar suas emoções.

\section{Estratégias de promoção de saúde}

A adolescência é um período marcado por profundas mudanças cognitivas, físicas, psicológicas e sociais, que orientam o jovem no desenvolvimento da autonomia. As alterações hormonais, maior aproximação com o grupo de amigos, busca pelo autoconhecimento e capacidade de desenvolver percepções críticas das situaçóes, reforçam a relevância da promoção de estratégias que favoreçam a saúde mental dos jovens estudantes ${ }^{6}$.

Diante deste cenário, foram planejadas ações que levassem a reflexões sobre temáticas relevantes, bem como a socialização entre pares, sendo mediadas pelos psicólogos escolares. Os momentos de escuta e orientações ocorreram de forma on-line e individual, com demandas pontuais e posterior encaminhamento para acompanhamento psicoterápico externo, caso identificado necessidade. Além disso, também foram realizadas escutas com grupos de alunos que apresentavam questôes semelhantes, visando orientar e consolidar a rede de apoio.

Foram desenvolvidas também palestras e dinâmicas, abordando temas como: neurociência e aprendizagem,organização de rotina, regulação do sono, autoimagem, luto, resiliência, medo e ansiedade, procrastinação, expectativas e esperança, histórias inspiradoras, direito constitucional nas escolas e mesas redondas das profissões. Todas as atividades elaboradastiveram por objetivo maior compreender o comportamento do alunado, identificar os sentimentos vivenciados, além de manter os jovens motivados e focados no seu projeto de vida, mesmo diante da atual crise vivenciada. Outro momento relevante foi a apresentação da atividade "cidadania e os protocolos sanitários", sendo este realizado em parceria com o serviço de enfermagem da escola.

\section{Estratégias do serviço de enfermagem}

Diante da demanda de saúde mental e especialmente de cuidados diante da pandemia do novo coronavírus (SARS-CoV-2), o enfermeiro é exigido a desenvolver competências específicas estabelecendo padrões profissionais, criando mecanismos para assegurar a qualidade do cuidado e ampliando conhecimentos e habilidades necessárias ${ }^{7}$.

Os protocolos sanitários adotados na escola são baseados nos estudos e pesquisas realizadas pela Organização Mundial de Saúde - OMS, bem como orientações emanadas pelo Ministério da Saúde, Agência Nacional de Vigilância Sanitária - ANVISA, Secretaria de Estado da Saúde do Piauí - SESAPI e o Comitê de Operações Emergenciais - COE. Todos os procedimentos discutidos nos órgãos e entidades mencionadas são trazidos para o ambiente escolar e postos em prática com um rigoroso controle de qualidade.

Um dos procedimentos adotados é a divisão do ambiente escolar por zonas (verde, amarela, vermelho) que indicam o risco biológico ali existente e a quantidade de pessoas que podem circular nesses espaços, bem como o tempo para permanência. A divisão em cores é a mesma adotada no ambiente hospitalar, sendo vermelho para alto risco, amarelo para médio risco e a cor verde é a indicativa de baixo risco de contaminação.

Não obstante a isto, são realizadas palestras e orientações aos alunos que estão frequentando a escola nas modalidades disponibilizadas (hibrido/remoto). Nas orientações levadas aos alunos pelo corpo de enfermagem é exposto de forma acessível e didática, a importância da obediência aos protocolos sanitários adotados na escola, bem como sobre o uso adequado dos equipamentos obrigatórios de segurança que todos devem possuir, tais como máscaras e álcool para higienização das mãos e objetos pessoais.

O corpo de enfermagem, ao se deparar com algum aluno com sintomas sugestivos de SARS-CoV-2, realiza contato com a família do adolescente para que o responsável compareça à escola e leve o aluno para que seja realizada uma avaliação médica mais 
detalhada sobre o seu estado de saúde.

Após isso, o setor de enfermagem fica acompanhando o caso diariamente, colhendo informações junto à família do adolescente para que possam ter informações atualizadas do seu quadro de saúde. Ressalte-se, todavia, que por cautela, o aluno é imediatamente posto no ensino remoto até que seja descartada qualquer possibilidade de infecção pelo SARS-CoV-2.

\section{DISCUSSÃO}

\section{O impacto da pandemia do novo co- ronavírus sobre a saúde mental dos adolescentes}

O novo coronavírus (SARS-CoV-2) ocasionou uma crise mundial que provocou uma série de mudanças e adaptações, afetando os mais diversos setores. No contexto educacional, é perceptível o impacto que a pandemia teve nos adolescentes, que se sentiram inseguros diante dessa readequação dos modelos educacionais. Nesse sentido, se faz importante esclarecer o conceito de adolescência, que corresponde a fase do desenvolvimento que ocorre entre a infância e a vida adulta ${ }^{8}$.

Vale ressaltar que é nesta fase que se intensifica o processo de individuação, sendo normalmente esperado um período de questionamento dos valores e um período importante referente às relações sociais e de amizade9. Em virtude disso, é comum o adolescente não se separar dos amigos mais próximos, gerando segurança emocional. Paralelo a isto, as medidas sanitárias de isolamento social, impossibilitaram os jovens de se relacionar fisicamente com os seus pares, além de impor um excesso de contato com a família, favorecendo a ausência de privacidade ${ }^{10}$.

Desta forma, o adolescente vivencia um processo de interrupção no seu percurso natural de busca por uma identidade, além de outros fatores como a insegurança na aprendizagem, alteração no sono e a intensa exposição a informações, que contribuem para o adoecimento do mesmo. Antes do início da pandemia, um a cada cinco estudantes tinham um ou mais transtornos mentais diagnosticáveis em todo mundo. Durante este período, com o fechamento das escolas e a interrupção abrupta do semestre letivo, os estudantes também passaram a vivenciar angústias e medos, o qual vem agravando os problemas de saúde mental entre os jovens ${ }^{11}$.

\section{As principais estratégias promotoras de saúde mental no contexto escolar em tempos de pandemia}

Diante dos inúmeros impactos causados em decorrência da pandemia, é notória a importância da escola reforçar a atenção para saúde mental da comunidade, em especial aos adolescentes, que como já exposto, vivenciam naturalmente uma fase de profundas mudanças biopsicossociais. É preciso que as escolas priorizem duas intervenções de promoção de saúde nesse período de pandemia, sendo estas, o desenvolvimento de estratégias para garantir o acesso ao serviço de saúde mental e a ampliação intencional desse serviço a estudantes em circunstâncias especiais ${ }^{12}$. Nesse sentido, embora a maioria das instituições ofereçam algum serviço de aconselhamento e apoio, é necessário adaptar e inovar para atender as especificidades dos alunos, a exemplo das práticas descritas anteriormente que foram realizadas pelo PEE e serviço de enfermagem.

É importante frisar que durante esse processo criativo relacionado ao ajustamento das funções acima citadas, as intervenções referentes aos serviços de saúde, são fundamentais para o tratamento de adolescentes com sofrimento psíquico anteriores a pandemia, evitando novas crises, bem como trabalhando de forma preventiva10. É durante esse período que o jovem tem que lidar com diversos fenômenos estressores, sendo que uma parcela destes podem lidar eficazmente com essas mudanças, enquanto outros não conseguem lidar, favorecendo o surgimento de algumas demandas de saúde mental ${ }^{13}$.

Em face a essa circunstância, a predominância de transtornos mentais é maior na população entre os 16 a 24 anos do que em outros períodos da vida, afetando $10 \%$ a $20 \%$ de jovens em todo mundo ${ }^{14}$. Uma das condições que naturalmente potencializam o surgimento do adoecimento mental durante essa faixa-etária está relacionada ao estresse escolar que geralmente provoca reaçôes psicológicas como ansiedade, raiva, depressão e confusão entre os alunos do ensino médio, tendo sido exacerbadas em face da pandemia de Covid-19 ${ }^{16}$.

Os alunos se viram obrigados a ficar em casa e a enfrentar novas pressóes de estudos, além de mudanças no ambiente e no estilo de ensino. Diante de tantas demandas que emergiram e ainda emergem, é fundamental utilizar de estratégias direcionadas para autorregulação emocional e aprendizagens. Nesse sentido, os educadores precisam estar atentos para as demandas subjetivas destes estudantes, especialmente as de cunho emocional, sendo importante discutir temas como "resiliência", que aumentam a capacidade de enfrentamento.

Da mesma forma, as escolas podem oferecer vivências em grupo virtuais, que podem ser um recurso acessível e escalonável. Embora a evidência de sua eficácia seja limitada, pois este não era um formato amplamente adotado antes da Covid-19, algumas observações iniciais mostram sua promessa. Essas estratégias são consistentes com as tendências nacionais mais amplas após o surto de Covid-19. Além disso, é relevante que os educadores estejam atentos às possibilidades individuais, assim como de cada esfera familiar ${ }^{13}$.

É imprescindível que professores reinventem e priorizem a saúde emocional, reformulando formas de ministrar o conteúdo escolar. Além disso, outras possíveis intervenções estão ligadas a acompanhamentos individuais de forma remota e manutenção do contato por telefone com adolescente e família ${ }^{16}$. Ademais, a arte,a cultura e atividades físicas são cruciais na promoção de saúde mental, especialmente dentre os adolescentes. Atividades que favoreçam a imaginação e a criatividade, que contribuam para percepção do jovem estudante sobre o sentimento de pertença a grupos pode favorecer o seu desenvolvimento saudável nesse momento ${ }^{17}$.

Portanto, constata-se que as estratégias relacionadas à promoção de saúde no 
contexto escolar, especialmente no atual cenário de crise, envolvem uma vigilância ainda mais intensa sobre os aspectos referentes à subjetividade dos adolescentes, de forma preventiva e remediativa, sendo necessário trabalhar temas relacionados ao fortalecimento pessoal destes, tais como resiliência, sentimento de pertencimento e o reconhecimento de suas potencialidades diante dos desafios apresentados.

Outrossim, campanhas relacionadas à saúde física e mental e o esclarecimento aos adolescentes sobre as medidas sanitárias adotadas pela escola em parceria com o setor de enfermagem, podem promover uma sensação maior de confiança e segurança dentro da instituição, que minimizem sentimentos potencialmente nocivos, como: ansiedade, insegurança e medo.

\section{CONCLUSÕES}

Ante o exposto no relato de experiência, observou-se queos objetivosforam alcançados. Ficou evidente que mesmo diante da situação pandêmica e das adversidades em relação às questões sociais e econômicas, a preocupação com a saúde e bem estar dos alunos é única e impulsionou para o desenvolvimento de novas ações.

As atividades que promovem saúde mental no contexto escolar são de suma importância. Percebe-se ao longo do tempo que o espaço escolar deixou de ser um ambiente meramente de aprimoramento cognitivo, contemplando também a formação de cidadãos e o desenvolvimento de habilidades socioemocionais, a partir da compreensão multidimencional que cada indivíduo apresenta. Dessa forma, o aluno deixou de ser visto como um mero reprodutor de conhecimento e passou a ser protagonista de seu aprendizado, entre outras coisas. $\mathrm{O}$ ambiente escolar oferece a ele autonomia, estimulando-o a buscar informação, trabalhar seu aspecto emocio- nal e a construir conhecimento caminhando com as próprias pernas.

Por fim, trabalhar temas tão relevantes com os adolescentes imersos nessa nova realidade ocasionada pela pandemia, demandaria maior trabalho de organização física e, talvez, contasse com menos participação presencial. Dessa maneira, observou-se que além de tempo dedicado, houve um olhar diferenciado e humanizado sobre as demandas expostas. E evidenciou a relevância de um fazer multiprofissional dentro da escola agregando saberes da psicologia e enfermagem em prol da promoção de saúde dentro do contexto escolar.

Porém, compreende-se que tais estratégias possuem suas limitações especialmente diante das mudanças rotineiras provocadas pelo atual cenário e por se perceber o indivíduo como esse ser histórico-cultural, evidenciando assim a importância de novos estudos sobre a problemática descrita no respectivo trabalho.

\section{REFERÊNCIAS}

1. Brasil. MEC/CONSED/UNDIME. Base Nacional Comum Curricu$\operatorname{lar}$ (BNCC). Educação é a Base. Brasília; 2017.

2. Casemiro JP, Fonseca ABC, Secco, FVM. Promover saúde na escola: reflexões a partir de uma revisão sobre saúde escolar na América Latina. Ciência \& Saúde Coletiva. 2014; 19(3): 829-840.

3. Menezes KM, Rodrigues CBC, Candito V, Soares FAA. Educação em Saúde no contexto escolar: construção de uma proposta interdisciplinar de ensino-aprendizagem baseada em projetos. Rev. Ed. Popular. 2020; 48-66.

4. Gerhardt TE, Silveira DT. Métodos de pesquisa. Porto Alegre: Editora UFRGS; 2009.

5. Araujo CMM, Almeida SFC. Psicologia Escolar:construção e consolidação da identidade profissional. São Paulo: Editora Alínea; 2014.

6. Eltink CF, Nunes CWB. Concepções sobre estresse segundo alunos do ensino médio de uma cidade de pequeno porte. REFACS. 2020; 8: 549-561.

7. Cássia TDA, Dias GA, Santos DBC, Cássia SPA, Prata MS, Cardoso ICC. Competências do enfermeiro no matriciamento em saúde mental: revisão integrativa. Saúde Coletiva. 2021; 63 (11).

8. Zagury T. O adolescente por ele mesmo: Como o jovem brasileiro vê a família, a escola, o lazer, a política, a profissão, o sexo, as drogas e a religião. Rio de Janeiro: Record; 2012.

9. Bohoslavsky R. Orientação Vocacional a estratégia clínica. São Paulo: Martins Fontes; 2007.
10. Miliauskas CR, Faus DP. Saúde mental de adolescentes em tempos de Covid-19: desafios e possibilidades de enfrentamento. Physis: Revista de Saúde Coletiva. 2020; 30 (4).

11. Zhai Y, Du X. Abordando a saúde mental universitária em meio à pandemia de Covid-19. 2020; 288: 113003.

12. Liu, CH. Prioridades para abordar o impacto da pandemia Covid-19 na saúde mental de estudantes universitários. Journalof American CollegeHealth.EstadosUnidos da América. 2020.

13. Lok NS, Lok N. "EfficiencyofPhysicalExerciseProgramsonChronicPsychiatryPatients: A SystematicReview". Psikiyatri de GuncelYaklasimlar- Current Approaches in Psychiatry. 2016; 8 (4).

14. Lök N, Bademli K, Canbaz M. The EffectsofAnger Management EducationonAdolescents' MannerofDisplayingAngerand Self-Esteem: A RandomizedControlledTrial; 2017.

15. Zhang Q, Zhou L, Xia J. Impacto do COVID-19 na resiliência emocional e gerenciamento de aprendizagem de alunos do ensino médio. Medical Science Monitor; 2020; 26.

16. Cruz NMLV, Souza EB, Sampaio CSF, Santos AJM, Chaves SV, Hora RN, et. al. Apoio psicossocial em tempos de COVID-19: experiências de novas estratégias de gestão e ajuda mútua no sul da Bahia, Brasil. APS emSaúde. 2020; 2 (2): 97-105.

17. Király O, Potenza MN, Steing DJ, King DL, Hodgins DC, Saunders $\mathrm{JB}$, et al. Preventingproblematic internet use duringthe COVID-19 pandemic: Consensus guidance. ComprehensivePsychiatry. 2020; 100: 152180. 